\title{
Therapeutic management of classic lichen planopilaris: a systematic review
}

This article was published in the following Dove Press journal:

Clinical, Cosmetic and Investigational Dermatology

\section{Enzo Errichetti \\ Matteo Figini \\ Margherita Croatto \\ Giuseppe Stinco}

Department of Experimental and Clinical Medicine, Institute of Dermatology, University of Udine, Udine, Italy
Correspondence: Enzo Errichetti Institute of Dermatology, "Santa Maria della Misericordia" University Hospital, Piazzale Santa Maria della Misericordia, 15, 33100 Udine, Italy

Tel +390432559822

Email enzoerri@yahoo.it

\begin{abstract}
Several treatment strategies have been proposed in classic lichen planopilaris (LPP), although no gold standard therapeutic approach has been recognized so far due to the variable and, sometimes, contradictory results reported in the literature, as well as due to the lack of guidelines and randomized controlled trials. In the present review, we sought to provide an updated overview on the treatment of classic LPP by analyzing the level of evidence of published studies, also proposing a possible therapeutic strategy according to the findings highlighted in this systematic review.
\end{abstract}

Keywords: lichen planopilaris, management, therapy, treatment

\section{Introduction}

Lichen planopilaris (LPP) is a relatively uncommon cutaneous disorder characterized by a chronic lymphocytic inflammation that leads to the selective destruction of hair follicles, thus resulting in scarring alopecia. ${ }^{1}$ Some authors consider LPP as a follicular form of lichen planus, although only about $30 \%$ of patients present cutaneous or mucosal lesions of lichen planus. ${ }^{2}$

LPP is more common in women than in men (ratio varying from 1.8:1 to 9:1), and the peak age of onset is observed between 30 and 60 years. ${ }^{1-4}$

Although pathogenesis of LPP is still poorly understood, many authors regard such a condition as a hair-specific autoimmune disorder in which T-lymphocytes target follicular antigens with the consequent destruction of the hair follicle stem cells. ${ }^{1-4}$ Possible involved inflammatory mediators include b-FGF and TGF- $\beta$, which would be responsible for fibroblast activation. ${ }^{1-3}$ Interestingly, recent evidence has pointed out a possible role of PPAR- $\gamma$ in the destruction of the pilosebaceous unit typical of LPP. ${ }^{3}$

LPP classically presents as follicular keratotic plugs and/or perifollicular scaling along with perifollicular erythema, with subsequent hair loss resulting in patchy alopecic areas. ${ }^{1,2}$ Of note, in acute phases, LPP patients may experience pruritus, pain, and/or burning sensation, differently from other primary scarring alopecias. ${ }^{1,2}$ Besides classic LPP, there are two main clinical variants, viz. frontal fibrosing alopecia and Graham-Little-Piccardi-Lasseur syndrome, with the former presenting with a progressing band of alopecia of the hairline in postmenopausal women and the latter being characterized by the triad of scarring patchy alopecia of the scalp, nonscarring alopecia of the axillae/pubic region, and spinous follicular papules of the trunk/limbs. ${ }^{1,3,4}$ 
The main differential diagnoses of LPP include discoid lupus erythematosus, alopecia areata, centrifugal cicatricial alopecia, and folliculitis decalvans. ${ }^{1-5}$ A good physical assessment, along with dermoscopic and histological examination, is important to distinguish LPP from such conditions. ${ }^{1-5}$

From a histological point of view, active lesions show a band-like subepidermal lymphocytic infiltrate, "hugging" the upper hair follicle (isthmus and infundibulum), with no involvement of the deeper portion of the follicle (differently from alopecia areata), while late lesions are mainly characterized by the reduction/loss of sebaceous glands and of arrector pili muscles, concentric perifollicular fibrosis, and irreversible destruction of the follicle with perifollicular hyalinization in both upper/lower dermis and follicular tract. ${ }^{2-4}$ Other specific histological features include mucinous perifollicular fibroplasia in the upper dermis, the absence of interfollicular mucin, and a superficial perifollicular wedge-shaped scarring. ${ }^{2-4}$ In $40 \%$ of cases, direct immunofluorescence shows colloid bodies and/or positive staining for immunoglobulin M (IgM) and, less commonly, IgA or C3; a linear band of fibrin and/or fibrinogen at the dermoepidermal junction may also be present..$^{2-4}$

The dermoscopy of LPP displays several features, with the most specific finding of active lesions being perifollicular scaling forming a sort of "collar" on the proximal portion of the hair shaft. Late lesions may show fibrotic white dots, acquired pili torti, loss of follicular openings, white areas, honeycomb/scattered hyperpigmentation, milky red areas, and hair tufts. ${ }^{5}$

Many treatment strategies have been proposed in classic LPP based on findings from anecdotal case reports, case series, or small studies. ${ }^{1-3}$ However, no gold standard therapeutic approach has been recognized so far due to the variable and, sometimes, contradictory results reported in the literature, as well as due to the lack of guidelines and randomized controlled trials. ${ }^{1-3}$ Besides, there is a lack of updated systematic reviews taking into account the level of evidence of treatment modalities for classic LPP. In this review, we sought to fill such a gap by providing an updated overview analyzing the level of evidence of published studies dealing with classic LPP therapies.

\section{Materials and methods}

All published information about LPP treatments was retrieved by a comprehensive search of the literature using the PubMed electronic database; the search term was "lichen planopilaris." A manual search was also carried out by analyzing the reference sections of all relevant studies or reviews about such a topic. All publications reporting the treatment of at least one classic LPP instance were considered, excluding frontal fibrosing alopecia, Graham-Little-Piccardi-Lasseur syndrome, and LPP exclusively involving areas other than scalp, as well as articles not specifying either therapeutic response outcome or LPP subtype. Notably, only English language papers were included in this review.

For each included study, reported variables such as author, year, the type of treatment, the type of study (classified according to standard definitions), ${ }^{6}$ the number of patients, and response outcomes were recorded. In addition, we also evaluated the level of evidence available for each considered paper, according to the most recent guidelines for evidencebased medicine, The Oxford 2011 Levels of Evidence: ${ }^{7}$ level of evidence I, systematic review of randomized trials or n-of-1 trials; II, randomized trial or observational study with dramatic effect; III, nonrandomized controlled cohort/followup study; IV, case series, case-control studies, or historically controlled studies; V, mechanism-based reasoning. Notably, single case reports were labeled as level of evidence V.

For practical purposes, we will first describe the treatments for which there is good evidence (if any) and then mention those having weaker evidence. In case of therapies having the same level of evidence, we will first list those with the greater number of treated patients.

\section{Results}

Table 1 summarizes all the results in detail. Importantly, all the following response rates refer to the proportion of patients experiencing objective clinical improvement regardless of the response degree (as it is not always mentioned in the various papers) and/or arrest of hair loss; isolated symptomatic improvement was not considered as a positive outcome. For details on the response degree, refer to Table 1.

\section{Hydroxychloroquine (highest level of evidence: Il; total number of patients: I27; global response rate: $5 \mathrm{I} .2 \%$ [65 of I27]; response rate in monotherapy: $51.0 \%$ [52 of 102])}

Several studies investigating the efficacy of antimalarials have been published, ${ }^{1,2,8-14}$ including a randomized clinical trial evaluating hydroxychloroquine ( $400 \mathrm{mg}$ daily) versus methotrexate (15 mg weekly) administered for 6 months in refractory LPP cases. ${ }^{8}$ In detail, although hydroxychloroquine yielded a significant Lichen Planopilaris Activity Index (LPPAI) decrease at months 2 and 4 (compared with baseline and month 2, respectively), such a study showed a higher efficacy for methotrexate, with a mean decrease in LPPAI 


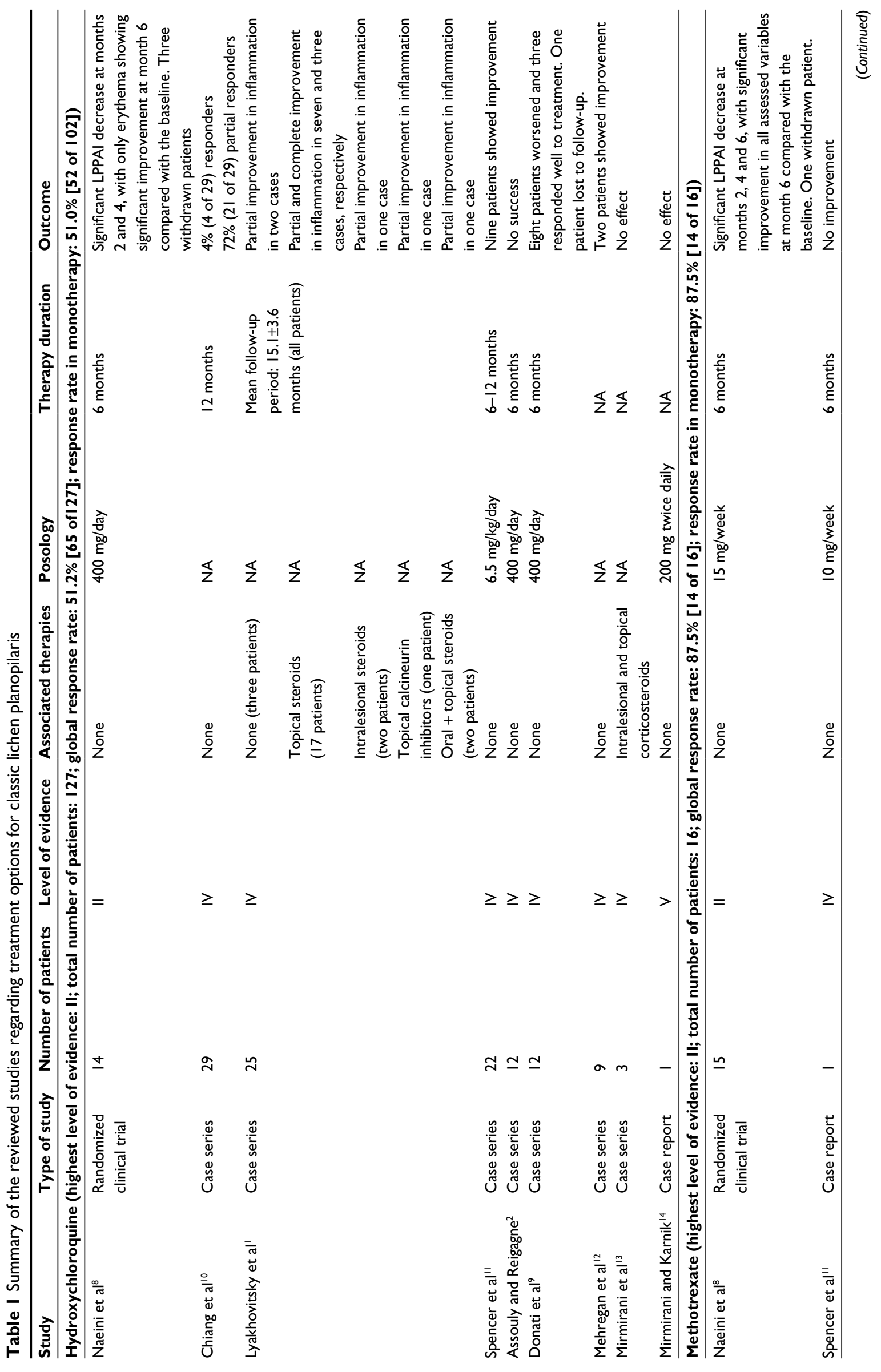




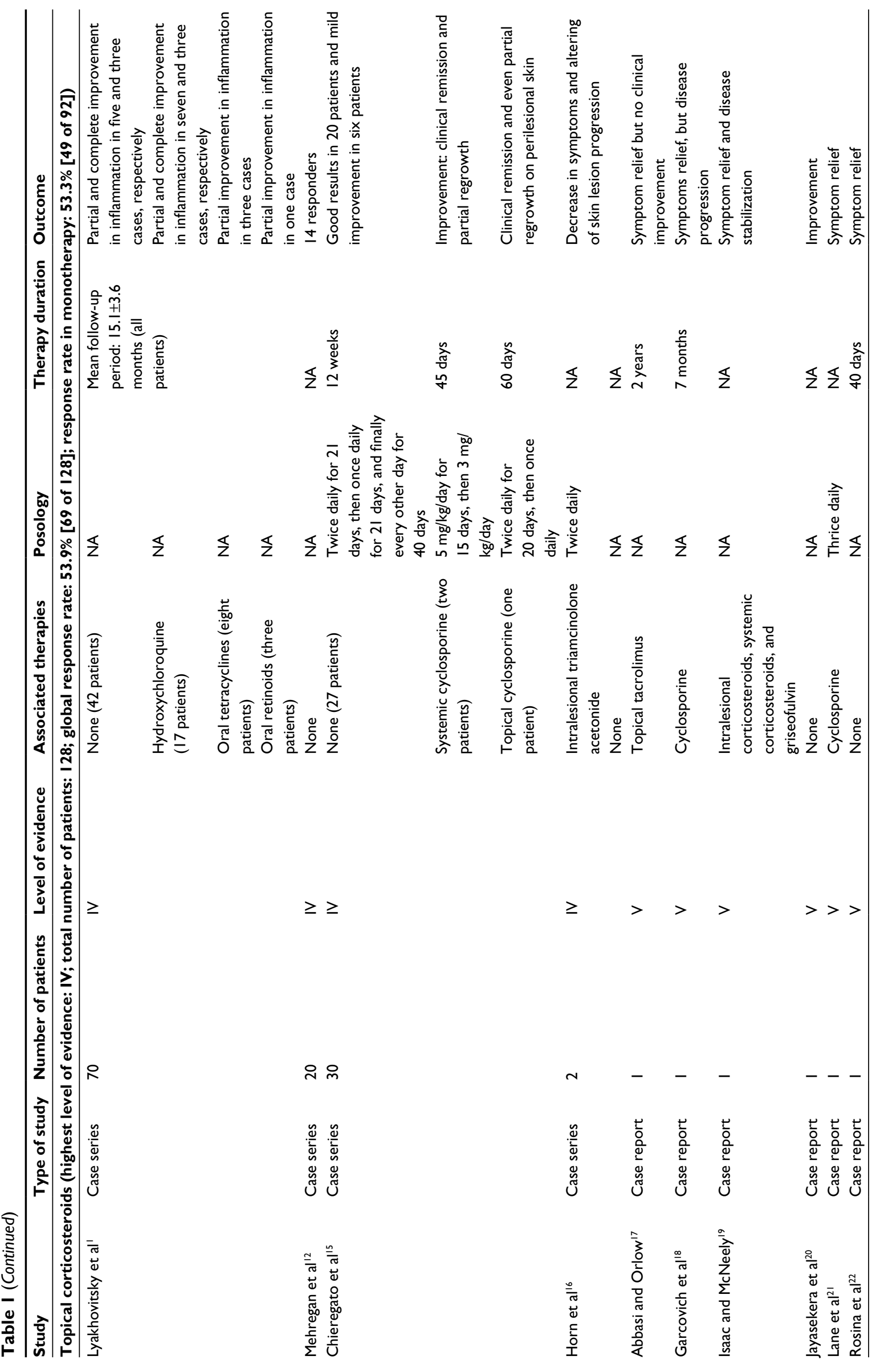




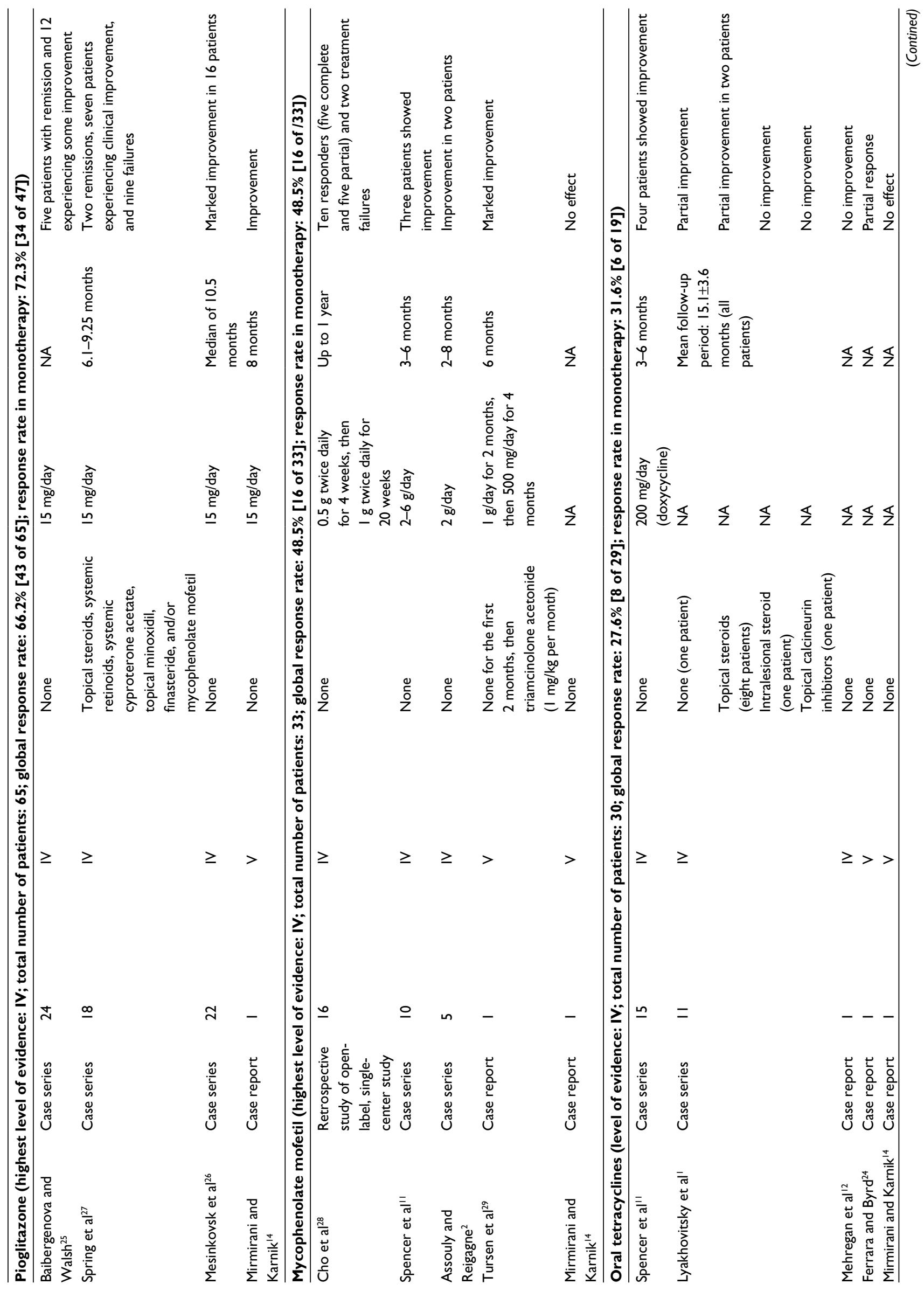




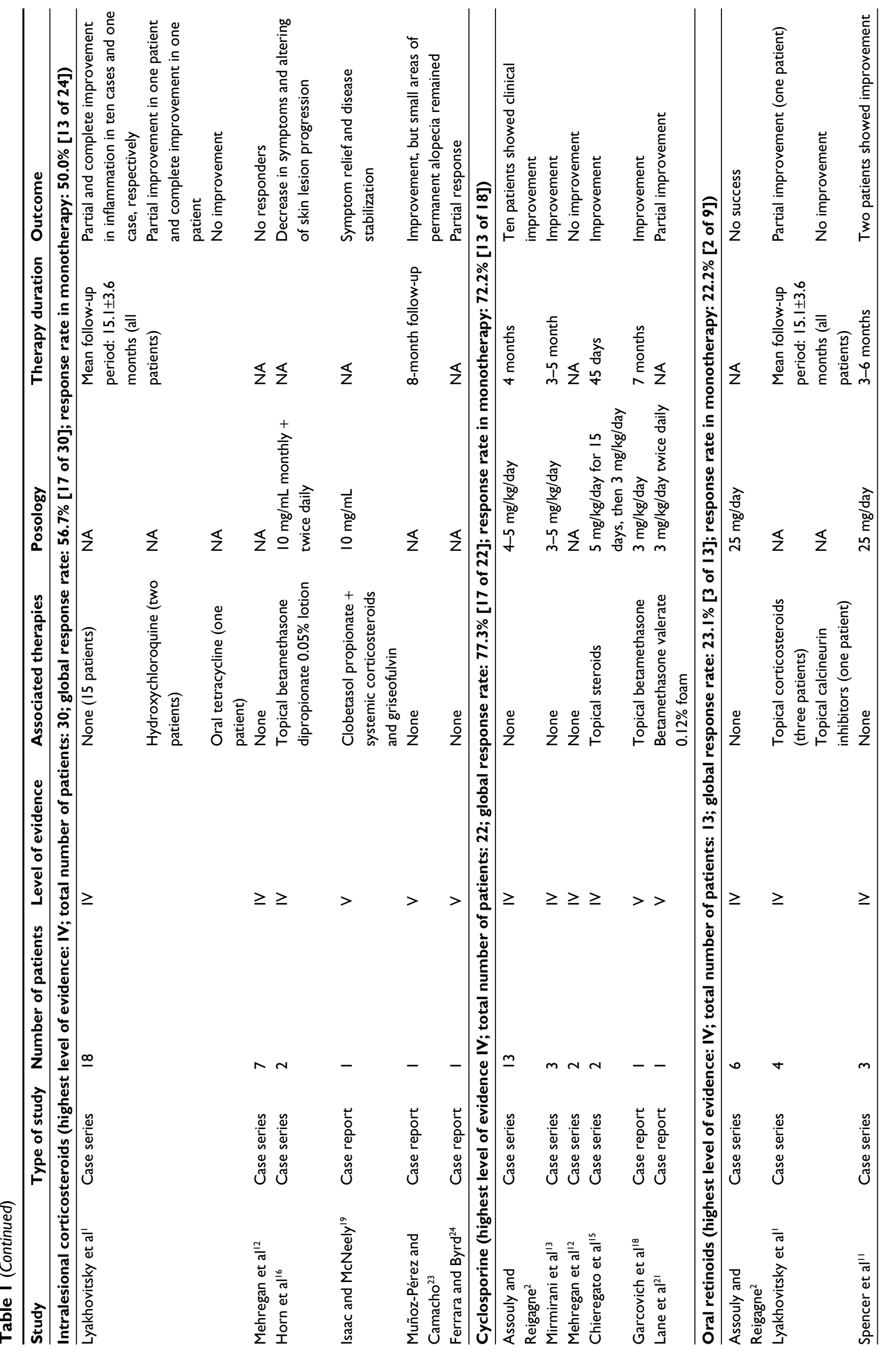




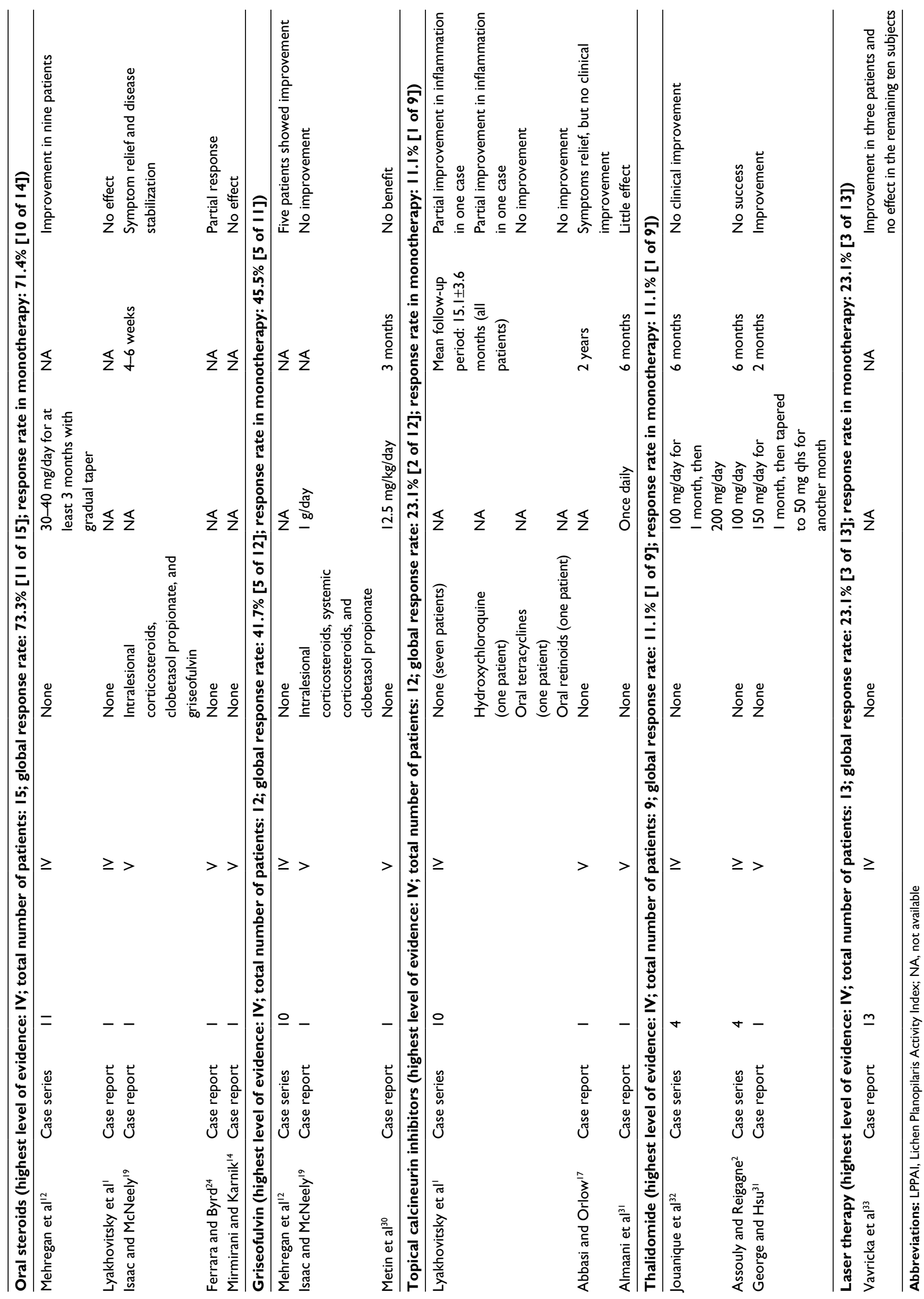


at the 6th month of $3.3 \pm 2.09$ versus $1.51 \pm 0.91(P=0.01) .^{8}$ Of note, in the hydroxychloroquine group, only erythema $(P=0.004)$ showed a significant improvement at the end of the study, while perifollicular erythema, perifollicular scaling, spreading, and follicular keratosis did not. ${ }^{8}$

Besides this comparative analysis, there are other studies on the use of antimalarials in LPP. ${ }^{1,2,9-14}$ In particular, a prospective study on 12 patients treated with hydroxychloroquine (400 mg daily) for 6 months found a good response in three cases (although their hair count was in a decreasing number) and progression in eight instances; one patient was lost during the follow-up. ${ }^{9}$ Higher success rates (including partial and complete responses) with the use of hydroxychloroquine were observed in other studies, with figures ranging from $40.1 \%$ to $76 \%$. $^{1,10,11}$ Conversely, other small case series or single case reports showed few results with the same drug, with little or no response. ${ }^{12-14}$

\section{Methotrexate (highest level of evidence:} II; total number of patients: 16; global response rate: $87.5 \%$ [ 14 of 16 ]; response rate in monotherapy: $87.5 \%$ [14 of 16])

The efficacy of methotrexate has mainly been studied in the abovementioned randomized clinical trial comparing hydroxychloroquine (400 mg daily) versus methotrexate (15 mg weekly) administered for 6 months. ${ }^{8}$ Apart from a higher global efficacy over hydroxychloroquine (see above), methotrexate also showed significant improvement in all the assessed variables, viz. pruritus $(P=0.007)$, erythema $(P=0.01)$, perifollicular erythema $(P=0.01)$, perifollicular scaling $(P=0.08)$, spreading $(P=0.001)$, and follicular keratosis $(P=0.04) .{ }^{8}$ Only a single LPP case showed no significant improvement with methotrexate. ${ }^{11}$

\section{Topical corticosteroids (highest level of evidence: IV; total number of patients:} I 28; global response rate: $53.9 \%$ [69 of 128]; response rate in monotherapy: $53.3 \%$ [49 of 92]) and intralesional corticosteroids (highest level of evidence: IV; total number of patients: 30; global response rate: $56.7 \%$ [ 17 of 30]; response rate in monotherapy: $50.0 \%$ [ 13 of 24])

Potent topical and intralesional (ie, triamcinolone acetonide) steroids are often among the first-line treatments in LPP. They have been used either in monotherapy or in association with other topical and/or systemic therapies, with variable degrees of success. ${ }^{1,12,15-24}$ In particular, Lyakhovitsky

et al reported a low success rate in patients treated with topical steroids (three complete responders and five partial responders in 42 patients treated in monotherapy), while they observed very good results when steroids were administered intralesionally (10 partial responders and one complete responder in 15 patients treated in monotherapy). ${ }^{1}$ Conversely, Mehregan et al observed a higher success rate in patients treated with topical steroids than those treated with intralesional steroids ( $70 \%$ [14 of 20$]$ versus $0 \%$ [0 of 7]). ${ }^{12}$ In addition, Chieregato et al found positive outcomes in subjects treated with topical corticosteroids, both alone or in association with systemic or topical cyclosporine (with an overall success rate of $93.3 \%-20$ of 30 "good results" and six of 30 "mild improvement"). ${ }^{15}$

Apart from the aforementioned studies, there are many reports describing one or few LPP patients undergoing topical and/or intralesional steroids, with very different results (from little-to-no improvement to good results with almost resolution of clinical features, with or without some degree of hair regrowth). ${ }^{16-24}$

\section{Pioglitazone (highest level of evidence:} IV; total number of patients: 65; global response rate: $66.2 \%$ [43 of 65]; response rate in monotherapy: $72.3 \%$ [34 of 47])

Pioglitazone (dose of pioglitazone: $15 \mathrm{mg} /$ day) has been reported as having encouraging results in LPP, with two studies reporting positive outcomes in the majority of patients, viz. five patients with remission and 12 experiencing some improvement in one analysis ${ }^{25}$ and marked improvement in 16 patients in the other study. ${ }^{26}$

Less positive findings were observed in a prospective observational study on 22 patients treated with pioglitazone along with another treatment (refer Table 1 for details), with two remissions, seven patients experiencing clinical improvement and nine experiencing failures. ${ }^{27}$

Symptoms relief and decrease in inflammation at 2-month and 6-month follow-ups were also observed in a multiresistant case treated with pioglitazone $(15 \mathrm{mg} /$ day $)$ for 8 months. $^{14}$

Mycophenolate mofetil (highest level of evidence: IV; total number of patients: 33; global response rate: $48.5 \%$ [ 16 of 33]; response rate in monotherapy: $48.5 \%$ [16 of 33])

Evidence from a retrospective chart analysis of an open-label trial including 16 LPP recalcitrant instances treated with 
mycophenolate mofetil ( $0.5 \mathrm{mg}$ twice daily for 4 weeks and then $1 \mathrm{~g}$ twice daily for at least 20 weeks) showed a complete response (reduction in baseline LPPAI $>85 \%$ ) in five patients, a partial response (reduction in baseline LPPAI ranging from $25 \%$ to $85 \%$ ) in a further five patients, and treatment failure (reduction in baseline LPPAI $<25 \%$ ) in two subjects; four patients withdrew from the study because of adverse events. ${ }^{28}$

Lower figures were observed in a retrospective study on 10 patients treated with mycophenolate mofetil (2-6 g/day for 3-6 months), with only $30 \%$ of them showing improvement. ${ }^{11}$ Similar success rate $(40 \%)$ was found in another study in which the drug was used at the dosage of $2 \mathrm{~g}$ daily for 2-8 months. ${ }^{2}$

Finally, a single case report described a complete remission with the use of mycophenolate mofetil at the dose of 500 mg twice daily for 6 months without recurrence at 3-month follow-up. ${ }^{29}$ No effect was observed in another case report. ${ }^{14}$

\section{Oral tetracyclines (level of evidence:} IV; total number of patients: 30; global response rate: $27.6 \%$ [ 8 of 29 ]; response rate in monotherapy: $31.6 \%$ [6 of 19])

In a retrospective study on 15 patients treated with oral doxycycline ( $200 \mathrm{mg}$ /day for 3-6 months in monotherapy), Spencer et al observed that four of 15 (27\%) subjects experienced positive results, while the rest of the cases had no improvement. ${ }^{11}$

Similar results were found by Lyakhovitsky et al, who observed that three of 11 patients treated with an unspecified oral tetracycline showed a partial response, whereas the other seven cases had no response. ${ }^{1}$

Three further single reports have been reported, with two instances showing failure ${ }^{12,14}$ and one case displaying partial response. ${ }^{24}$

\section{Cyclosporine (highest level of evidence} IV; total number of patients: 22; global response rate: $77.3 \%$ [ 17 of 22 ]; response rate in monotherapy: $72.2 \%$ [13 of 18])

Cyclosporine is another common treatment for LPP.,12,13,15,18,21 A small prospective study on 13 subjects treated with oral cyclosporine (4-5 mg/kg/day for 4-6 months) showed clinical response in 10 cases; relapse rate was between $60 \%$ and $80 \%$, respectively, 6 months and 12 months after treatment discontinuation. $^{2}$

In addition, several other small case series and single case reports on the use of oral cyclosporine have been published, with most of them showing good outcomes, but also a significant likelihood of relapse after treatment discontinuation. . $^{12,13,15,18,21}$

Oral retinoids (highest level of evidence: IV; total number of patients: I 3; global response rate: $23.1 \%$ [3 of I3]; response rate in monotherapy: $22.2 \%$ [2 of 9])

Three small case series have investigated the effect of oral retinoids on classic LPP, with three of a total of 13 patients displaying positive outcomes. ${ }^{1,2,11}$

\section{Oral steroids (highest level of evidence:} IV; total number of patients: I5; global response rate: $73.3 \%$ [II of I5]; response rate in monotherapy: $71.4 \%$ [ 10 of 14])

Results with oral steroids are generally good, ${ }^{1,12,14,19,24}$ with the largest study (11 patients) investigating their efficacy in LPP showing a success rate of $82 \% .{ }^{12}$ However, it is also true that the likelihood of relapsing is very high, with $80 \%$ of patients experiencing a relapse within 1 year after drug withdrawal. ${ }^{12}$ Such a trend is confirmed by single case reports reported in the literature. ${ }^{1,14,19,24}$

\section{Griseofulvin (highest level of evidence:} IV; total number of patients: I2; global response rate: $41.7 \%$ [5 of 12 ]; response rate in monotherapy: $45.5 \%$ [5 of $\mathrm{II}]$ ) In a study on ten LPP patients treated with oral griseofulvin (dose, frequency, and treatment duration not specified), Mehregan et al observed that $50 \%$ of cases showed improvement. ${ }^{12}$

Two further LPP instances treated with griseofulvin have been reported, with no significant results in monotherapy ${ }^{30}$ or in association with other treatments. ${ }^{19}$

Topical calcineurin inhibitors (highest level of evidence: IV; total number of patients: I2; global response rate: $23.1 \%$ [2 of I2]; response rate in monotherapy: II.1\% [I of 9])

Results with topical calcineurin inhibitors are generally disappointing, with the largest study (ten patients) dealing with the usefulness of such a therapy in LPP displaying partial improvement in inflammation in only two cases (one in monotherapy and one associated with hydroxychloroquine). ${ }^{1}$ Besides this study, there are also another two case reports 
about the use of topical calcineurin inhibitors in LPP, showing little improvement. ${ }^{17,31}$

\section{Thalidomide (highest level of evidence: IV; total number of patients: 9; global response rate: II.I\% [I of 9]; response rate in monotherapy: II.I\% [I of 9])}

Although positive outcomes have been described in a single case report, ${ }^{32}$ two case series, respectively, involving four patients (each) showed no significant improvement with a dose of thalidomide of $100 \mathrm{mg} /$ day for 6 months $^{2}$ or 100 $\mathrm{mg}$ /day for 1 month and then $200 \mathrm{mg}$ /day for a further 6 months. $^{33}$

\section{Laser therapy (highest level of evidence:} IV; total number of patients: I 3; global response rate: $23.1 \%$ [3 of 13]; response rate in monotherapy: $23.1 \%$ [3 of I3])

A case series of 13 patients treated with 308-nm excimer laser showed an improvement in three patients and no effect in the remaining 10 subjects. $^{34}$

\section{Discussion}

Therapeutic aims in LPP mainly consist of reducing possible associated symptoms and halting disease activity, thereby preventing the development of further alopecic areas. ${ }^{1,2}$ However, being a relatively rare disease, literature data on the treatment of LPP are quite sparse, and no gold standard approach exists. ${ }^{1,2}$ Consequently, LPP treatment in daily clinical practice often relies on physician's personal experience, although some authors have proposed possible therapeutic strategies. ${ }^{1,2}$ In particular, topical steroids are often reported as a first-line treatment (particularly for limited cases), especially the ultrapotent corticotherapy clobetasol propionate. $^{2}$ A proposed protocol consists of using such a type of topical steroid twice daily for the first month, followed by an application once a day for 3 months, and then every other day for 3 more months. ${ }^{2}$ Although some authors have advocated the use of systemic oral corticosteroid therapy as a second-line treatment (prednisone $1 \mathrm{mg} / \mathrm{kg} /$ day for 15 days, tapered over 4 months), the very high degree of relapse (around $80 \%$ of patients) after treatment suspension makes such a therapy little useful in the long-term period. ${ }^{2}$ For this reason, other authors suggested to administer oral hydroxychloroquine (usually $200 \mathrm{mg}$ twice daily) as initial systemic therapy, which may be switched to cyclosporine (3-5 mg/ $\mathrm{kg} / \mathrm{d}$ ) if manifestations continue after 2-4 months of treatment. ${ }^{1,2}$ However, cyclosporine is commonly characterized by both a high relapse rate $(60 \%-80 \%$ after $6-12$ months from withdrawal) and relevant side effects over a long-term period. ${ }^{1,2}$ Because of such reasons, mycophenolate mofetil has been proposed as a possible and preferable alternative to cyclosporine due to the safer adverse effect profile. ${ }^{2}$ For recalcitrant LPP instances, other therapies have been considered, including oral retinoids, oral tetracycline, methotrexate, griseofulvin, thalidomide, laser therapy, topical calcineurin inhibitors, and pioglitazone. ${ }^{1,2}$

Importantly, the abovementioned treatment strategies are not the result of evidence-based therapeutic guidelines, thus making their validity quite questionable. In fact, according to the present review, there is only one study with a high level of evidence, namely a randomized clinical trial (level of evidence: II) comparing hydroxychloroquine and methotrexate for a 6-month period in recalcitrant LPP. Interestingly, this study revealed not only a significant superiority of methotrexate over hydroxychloroquine, but also the very limited response of recalcitrant LPP to the latter medication (efficacy only on erythema degree), differently from methotrexate which showed efficacy on pruritus as well as on all the objective variables assessed in the study (erythema, perifollicular erythema, perifollicular scaling, spreading, and follicular keratosis). However, it is noteworthy to emphasize that the use of hydroxychloroquine in LPP is not always unsuccessful as there are several reports showing positive results, with a response rate in monotherapy of $51.0 \%$ considering all the cases reported in the literature. It is possible to speculate that the negative outcomes observed in the abovementioned clinical trial could be due to the fact that it was focused only on recalcitrant cases.

According to our review, the efficacy of other commonly used/suggested therapies, including topical/intralesional/ oral steroids, oral cyclosporine, and oral mycophenolate mofetil, is based only on studies with low level of evidence (case series and case reports - level of evidence: IV). Such therapies have been reported to be useful in classic LPP, with an overall response rate in monotherapy of $53.3 \%$, $50.0 \%, 71.4 \%, 72.2 \%$, and $48.5 \%$ for topical steroids, intralesional steroids, oral steroids, oral cyclosporine, and oral mycophenolate mofetil, respectively. Obviously, topical/intralesional steroids are more suitable for cases with limited involvement, while oral steroids, oral cyclosporine, and oral mycophenolate mofetil are commonly suggested for extensive forms. However, as previously stated, use of 
both oral steroids and cyclosporine are characterized by a high relapse rate after their suspension as well as significant side effects in the case of prolonged administration, thus making oral mycophenolate mofetil a better choice over a long-term period.

Similarly, the level of evidence available for all the other treatments reported in the literature is low (case series and/or single case reports - level of evidence: IV/V), with the following response rates (in monotherapy): $31.6 \%$ for oral tetracyclines, $72.3 \%$ for pioglitazone, $23.1 \%$ for laser therapy, $22.2 \%$ for oral retinoids, $45.5 \%$ for griseofulvin, $11.1 \%$ for topical calcineurin inhibitors, and $11.1 \%$ for thalidomide.

Based on previously suggested therapeutic strategies, drug safety profiles/manageability, and the level of evidence/success rates highlighted in this systematic review, it is possible to speculate that topical/intralesional steroids and hydroxychloroquine might be a reasonable first-line therapy in localized and extensive classic LPP cases, respectively. In the case of topical/intralesional steroids resistance and progressive course, patients with localized forms may be switched to hydroxychloroquine. When experiencing therapy failure with hydroxychloroquine, methotrexate could be used as a second-line therapy, while mycophenolate mofetil and cyclosporine could be considered as third-line therapies, with the first one to be preferred over a long-term period because of the safer adverse effect profile with prolonged use. In our opinion, a short course of systemic steroids should be considered only to halt the progression and to improve symptoms in rapidly progressive and severe cases. When necessary, topical/intralesional steroids may be added to systemic therapies in the case of persistence of limited active areas. Interestingly, according to the results highlighted in this review, pioglitazone could be a promising and effective therapeutic option, although more evidence is needed to confirm its precise role in the LPP management. Based on available levels of evidence and success rates, we believe it could be considered as a third-line treatment, beside cyclosporine and mycophenolate mofetil. Figure 1 summarizes the proposed treatment strategy.

Of note, it has to be kept in mind that the abovementioned therapeutic management is not the result of head-to-head comparisons, and treatment outcomes reported in the various studies are quite variable. Therefore, it should be viewed with a critical eye and regarded as general advice which has to be adapted on case-by-case basis. Future randomized and controlled prospective studies are needed to better define the optimal therapeutic approach in LPP.

\section{Disclosure}

The authors report no conflicts of interest in this work.

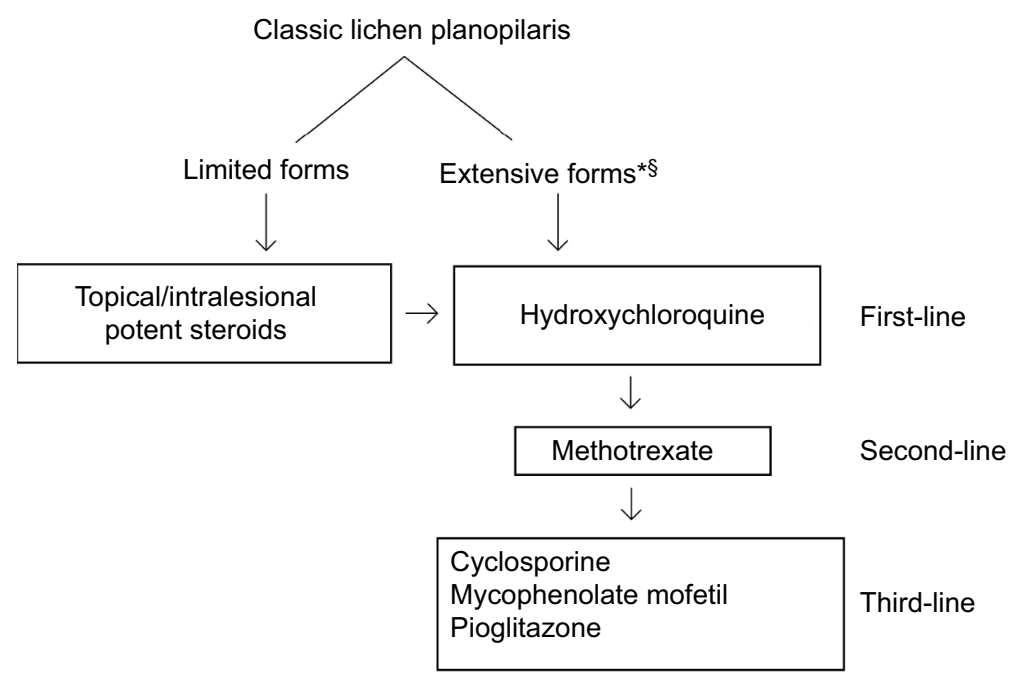

Figure I Proposed treatment strategy for classic lichen planopilaris.

Notes: *Topical/intralesional steroids may be added to systemic therapies in the case of persistence of limited active areas. ${ }^{\S} \mathrm{A}$ short course of systemic steroids should be considered only to halt the progression and to improve symptoms in rapidly progressive and severe cases. 


\section{References}

1. Lyakhovitsky A, Amichai B, Sizopoulou C, Barzilai A. A case series of 46 patients with lichen planopilaris: demographics, clinical evaluation, and treatment experience. J Dermatolog Treat. 2015;26(3):275-279.

2. Assouly P, Reigagne P. Lichen planopilaris: update on diagnosis and treatment. Semin Cutan Med Surg. 2009;28(1):3-10.

3. Rácz E, Gho C, Moorman PW, Noordhoek Hegt V, Neumann HA. Treatment of frontal fibrosing alopecia and lichen planopilaris: a systematic review. J Eur Acad Dermatol Venereol. 2013;27(12):1461-1470.

4. Piguet V, Breathnach SM, Le Cleach L. Lichen planus and lichenoid disorders. In: Griffiths CEM, Barker J, Bleiker T, Chalmers R, Creamer D, editors. Rook's Textbook of Dermatology. 9th ed. Oxford: WileyBlackwell; 2016:37.6-37.7.

5. Errichetti E, Stinco G. Dermoscopy in general dermatology: a practical overview. Dermatol Ther (Heidelb). 2016;6(4):471-507.

6. Porta M. A Dictionary of Epidemiology. 6th ed. New York: Oxford University Press; 2014.

7. Oxford Centre for Evidence-Based Medicine. The Oxford 2011 Levels of Evidence. Available from: http://www.cebm.net/index.aspx?o=5653. Accessed December 9, 2017.

8. Naeini FF, Saber M, Asilian A, Hosseini SM. Clinical efficacy and safety of methotrexate versus hydroxychloroquine in preventing lichen planopilaris progress: a randomized clinical trial. Int J Prev Med. 2017;8:37.

9. Donati A, Assouly P, Matard B, Jouanique C, Reygagne P. Clinical and photographic assessment of lichen planopilaris treatment efficacy. $\mathrm{J} \mathrm{Am}$ Acad Dermatol. 2011;64(3):597-598.

10. Chiang C, Sah D, Cho BK, Ochoa BE, Price VH. Hydroxychloroquine and lichen planopilaris: efficacy and introduction of Lichen Planopilaris Activity Index scoring system. J Am Acad Dermatol. 2010;62(3):387-392.

11. Spencer LA, Hawryluk EB, English JC 3rd. Lichen planopilaris: retrospective study and stepwise therapeutic approach. Arch Dermatol. 2009;145(3):333-334.

12. Mehregan DA, Van Hale HM, Muller SA. Lichen planopilaris: clinical and pathologic study of forty-five patients. J Am Acad Dermatol. 1992;27(6 Pt 1):935-942.

13. Mirmirani P, Willey A, Price VH. Short course of oral cyclosporine in lichen planopilaris. J Am Acad Dermatol. 2003;49(4):667-671.

14. Mirmirani P, Karnik P. Lichen planopilaris treated with a peroxisome proliferator-activated receptor gamma agonist. Arch Dermatol. 2009;145(12):1363-1366.

15. Chieregato C, Zini A, Barba A, Magnanini M, Rosina P. Lichen planopilaris: report of 30 cases and review of the literature. Int J Dermatol. 2003;42(5):342-345.

16. Horn RT Jr, Goette DK, Odom RB, Olson EG, Guill MA. Immunofluorescent findings and clinical overlap in two cases of follicular lichen planus. J Am Acad Dermatol. 1982;7(2):203-207.
17. Abbasi NR, Orlow SJ. Lichen planopilaris noted during etanercept therapy in a child with severe psoriasis. Pediatr Dermatol. 2009;26(1):118.

18. Garcovich S, Manco S, Zampetti A, Amerio P, Garcovich A. Onset of lichen planopilaris during treatment with etanercept. $\mathrm{Br} J$ Dermatol. 2008;158(5):1161-1163.

19. Isaac M, McNeely MC. Dermatitis herpetiformis associated with lichen planopilaris. J Am Acad Dermatol. 1995;33(6):1050-1051.

20. Jayasekera PS, Walsh ML, Hurrell D, Parslew RA. Case report of lichen planopilaris occurring in a pediatric patient receiving a tumor necrosis factor $\alpha$ inhibitor and a review of the literature. Pediatr Dermatol. 2016;33(2):e143-e146.

21. Lane TK, Kamino H, Walters RF, Meehan S, Pomeranz MK. Lichen planopilaris and psoriasis. Dermatol Online J. 2008;14(10):4.

22. Rosina P, Chieregato C, Magnanini M, Barba A. Lichen planopilaris and autoimmune thyroiditis. J Eur Acad Dermatol Venereol. 2002;16(6):648-649.

23. Muñoz-Pérez MA, Camacho F. Lichen planopilaris and scleroderma en coup de sabre. J Eur Acad Dermatol Venereol. 2002;16(5):542-544.

24. Ferrara RJ, Byrd RC. Lichen planopilaris: (follicular lichen planus). Cutis. 1973;12:869-870.

25. Baibergenova A, Walsh S. Use of pioglitazone in patients with lichen planopilaris. J Cutan Med Surg. 2012;16(2):97-100.

26. Mesinkovska NA, Tellez A, Dawes D, Piliang M, Bergfeld W. The use of oral pioglitazone in the treatment of lichen planopilaris. $J$ Am Acad Dermatol. 2015;72(2):355-356

27. Spring P, Spanou Z, de Viragh PA. Lichen planopilaris treated by the peroxisome proliferator activated receptor- $\gamma$ agonist pioglitazone: lack of lasting improvement or cure in the majority of patients. $J$ Am Acad Dermatol. 2013;69(5):830-832.

28. Cho BK, Sah D, Chwalek J, et al. Efficacy and safety of mycophenolate mofetil for lichen planopilaris. J Am Acad Dermatol. 2010;62(3):393-397.

29. Tursen U, Api H, Kaya T, Ikizoglu G. Treatment of lichen planopilaris with mycophenolate mofetil. Dermatol Online J. 2004;10(1):24.

30. Metin A, Calka O, Ugras S. Lichen planopilaris coexisting with erythema dyschromicum perstans. Br J Dermatol. 2001;145(3):522-523.

31. Almaani N, Liu L, Perez A, Robson A, Mellerio JE, McGrath JA. Epidermolysis bullosa pruriginosa in association with lichen planopilaris. Clin Exp Dermatol. 2009;34(8):e825-e828.

32. George SJ, Hsu S. Lichen planopilaris treated with thalidomide. J Am Acad Dermatol. 2001;45(6):965-966.

33. Jouanique $\mathrm{C}$, Reygagne P, Bachelez H, Dubertret L. Thalidomide is ineffective in the treatment of lichen planopilaris. J Am Acad Dermatol. 2004;51(3):480-481.

34. Vavricka BP, Haug I, Eliades I, Trueb R. 308-nm excimer laser treatment of lichen planopilaris of the scalp. Dermatology. 2006;213:74.
Clinical, Cosmetic and Investigational Dermatology

\section{Publish your work in this journal}

Clinical, Cosmetic and Investigational Dermatology is an international, peer-reviewed, open access, online journal that focuses on the latest clinical and experimental research in all aspects of skin disease and cosmetic interventions. This journal is included on PubMed. The manuscript management system is completely online
Dovepress

and includes a very quick and fair peer-review system, which is all easy to use. Visit http://www.dovepress.com/testimonials.php to read real quotes from published authors 